\title{
Reduction of Discrete Dynamical Systems with Applications to Dynamics Population Models
}

\author{
R. Bravo de la Parra ${ }^{*}$, M. Marvá ${ }^{1}$, E. Sánchez ${ }^{2}$, L.Sanz $^{2}$ \\ ${ }^{1}$ Departamento de Física y Matemáticas, Universidad de Alcalá \\ 28871 Alcalá de Henares (Madrid), Spain \\ 2 Departamento de Matemática Aplicada, ETSI Industriales \\ Universidad Politécnica de Madrid \\ José Gutiérrez Abascal 2, 28006 Madrid, Spain
}

\begin{abstract}
In this work we review the aggregation of variables method for discrete dynamical systems. These methods consist of describing the asymptotic behaviour of a complex system involving many coupled variables through the asymptotic behaviour of a reduced system formulated in terms of a few global variables. We consider population dynamics models including two processes acting at different time scales. Each process has associated a map describing its effect along its specific time unit. The discrete system encompassing both processes is expressed in the slow time scale composing the map associated to the slow one and the k-th iterate of the map associated to the fast one. In the linear case a result is stated showing the relationship between the corresponding asymptotic elements of both systems, initial and reduced. In the nonlinear case, the reduction result establishes the existence, stability and basins of attraction of steady states and periodic solutions of the original system with the help of the same elements of the corresponding reduced system. Several models looking over the main applications of the method to populations dynamics are collected to illustrate the general results.
\end{abstract}

Keywords and phrases: discrete models, aggregation of variables, time scales, population dynamic.

Mathematics Subject Classification: 39A11, 92D25

\section{Introduction}

Mathematical models used in ecology often inherit the complexity found in natural systems. They are governed by a large number of variables corresponding to various interacting organization levels. Methods of reduction and approximation are then used in order to transform such models into mathematically tractable ones. An important class of such methods are the so-called aggregation of variables methods. They are well adapted to deal with the problem of up-scaling in the context of ecological hierarchy theory [19].

The term aggregation of variables appeared first in economy and later introduced in ecology [16, 20]. The aggregation of a system consists of finding a certain number of global variables, functions of its

${ }^{*}$ Corresponding author. E-mail: rafael.bravo@uah.es 
state variables, and a system describing their dynamics. Aggregation is called perfect if the dynamics of the global variables is identical both in the initial system and in the aggregated one [16]. As perfect aggregation is a very infrequent issue to be found, a number of approximate aggregation techniques, [17], have been developed. Approximate aggregation deals with methods of reduction where the consistency between the dynamics of the global variables in the initial and the aggregated systems is only approximate. The study of aggregation methods linked to the existence of different time scales were initiated in 1989 by P. Auger [2], in the frame of ordinary differential equations. The method was refined and justified in terms of an adequate version of the centre manifold theorem [14] in [7]. Since then these methods have been extended into different mathematical settings [3, 4,6,28].

In this work we review the aggregation of variables method for discrete dynamical systems. The first attempts to extend the method to the setting of deterministic discrete dynamical systems date back to 1995. In [8] a very general linear discrete model with two time scales is aggregated and it is proved that the elements defining the asymptotic behaviour of the general system are approximated by those of the aggregated system. When two different processes acting at different time scales are included in the same discrete model it is necessary to choose the appropriate time unit. This choice, in contrast with continuous dynamical systems, is crucial since we cannot perform a change of the time variable that yields an equivalent system. In [8] the time unit of the system is chosen to be that of the fast process and the effect of the slow one is approximated for a fast time unit. In applications this choice is not always feasible because the action of the slow process during a fast time unit might not be describable. Let us think, for instance, of a population with separate generations, the process of reproduction cannot be represented if we choose a time unit smaller than the time elapsed between breeding seasons. Using the time unit associated to the slow dynamics, in [27] a similar linear system to that of [8] is treated, obtaining analogous asymptotic results. When the system is expressed in the slow time unit to describe the action of the fast process we only need to repeat it a large enough number of times.

The aggregation results in $[8,27]$ are applied to models of structured populations. In [30] the results for the slow time unit case of [27] are generalized. Further generalizations to non-autonomous and stochastic linear discrete systems are found in $[29,31,32]$. In [10] there is the first approach to aggregate a class of nonlinear discrete systems. They involve two time scales and they are expressed in the fast time unit. Considering the fast dynamics to be linear, a version of the center manifold theorem is applied to build up the aggregated system in a similar way to that used in the case of ordinary differential equations. This procedure is not valid for systems expressed in the slow time unit. A first attempt to overcome the problem is done in [9], where the slow dynamics is linear, and thus represented by a general nonnegative matrix, while the fast dynamics is dependent on global variables and is supposed to act a large number of times during one single time unit of the slow dynamics.

The aim of this work is to review the main results of aggregation of variables in discrete systems, both linear and nonlinear, expressed in the slow time unit. To build up this kind of systems in the frame of population dynamics we first think of two different processes acting at different time scales. The effect of the fast process during a fast time unit is represented by a general map $F$, analogously a map $S$ describes the slow process in the slow time unit. The combined effect of both processes during a slow time unit is then represented as the composition of map $S$ and the k-th iterate of map $F$, assuming that the slow time unit is divided into a large number $k$ of fast time units. Denoting by vector $X$ the state variables and $n$ the slow time variable, the general form of the system is:

$$
X_{k, n+1}=S\left(F^{k}\left(X_{k, n}\right)\right) .
$$

We present its approximate aggregation in a simple abstract form. We assume that there exists a limit system for $k$ tending to infinity that admits perfect aggregation. To ensure the perfect aggregation of the limit system we generalize the conditions to hold in the linear case. A linear discrete system can be perfectly aggregated whenever the associated matrix of order $N$ can be written as the product of two matrices of dimensions $N \times q$ and $q \times N$, respectively, with $q<N$. If this is the case a new system with $q$ variables can be defined and the dynamics of both systems can be obtained from each other. The 
translation into the nonlinear case requires that the associated map can be expressed as the composition of two maps going through a less dimensional space.

Assuming the perfect aggregation of the limit system, in the linear case it suffices the simple convergence of the sequence of matrices (Theorem 2.5) to establish the relationship between the corresponding asymptotic elements of both systems initial and aggregated. In the nonlinear case, it is stated in Theorem 2.6 how it is possible to study the existence, stability and basins of attraction of steady states and periodic solutions of the original system performing the study for the corresponding aggregated system. The hypotheses of Theorem 2.6 include the convergence on compact sets of the sequence of maps and their differentials. This latter assumption is generally difficult to be proved in particular applications.

The organization of the paper is as follows: in Section 2 a general discrete model that contains as a particular case the model (1.1) is introduced. The aggregation of variables procedure is developed, stating the aforementioned Theorems 2.5 and 2.6 that encompass the aggregation results for linear and nonlinear systems respectively. The particularization of these results to models of population dynamics of the form (1.1) is carried out in Section 3. Section 4 is devoted to applications. Section 4.1 illustrates the aggregation of linear discrete systems by means of a simple model of a population structured into juvenile and adult classes and which individuals migrate between two patches. Two different cases are treated: the first one considers migrations fast compared to demography and the second one re-scales deaths at the fast time scale. There are three applications of the aggregation of nonlinear discrete systems. They present three different situations where the cumbersome hypotheses of Theorem 2.6 are proved. In all three applications the population is supposed to be divided into groups and each of these groups into several subgroups. The fast dynamics is assumed internal for each group, conservative of a global variable, usually the total number of individuals, and with an asymptotically stable distribution among the subgroups. In Section 4.2 the fast dynamics for each group is represented by a projection matrix which is a regular stochastic matrix depending on the total number of individuals in each group. In Section 4.3 the fast dynamics is defined by projection matrices of two different kinds: for a first set of groups the matrices are constant whereas those of the second group depend on the densities of the subgroups associated to the groups in the first set. Finally, in Section 4.4 a model where fast dynamics is not described through a matrix is presented. The paper ends with a section including conclusions and some perspectives of future work.

\section{Reduction of discrete dynamical systems.}

In this section we introduce a general class of discrete dynamical systems that admit an approximate reduction. The reduction procedure is presented for both linear and nonlinear systems.

We start by introducing the original or complete system defined by

$$
X_{k, n+1}=H_{k}\left(X_{k, n}\right),
$$

where $k \in \mathbb{N}$ and, for $\Omega_{N} \in \mathbb{R}^{N}$ nonempty open set, $H_{k}: \Omega_{N} \rightarrow \Omega_{N}$ and $H_{k} \in C^{1}\left(\Omega_{N}\right)$.

To be able to build a reduced system, we assume the following conditions:

Hypothesis 2.1. The sequence of maps $\left\{H_{k}\right\}_{k \in \mathbb{N}}$ converges pointwise in $\Omega_{N}$ to a map $H: \Omega_{N} \rightarrow \Omega_{N}$ such that $H \in C^{1}\left(\Omega_{N}\right)$.

Hypothesis 2.2. There exist a non-empty open subset $\Omega_{q} \subset \mathbb{R}^{q}$ with $q<N$ and two maps $G: \Omega_{N} \longrightarrow$ $\Omega_{q}$ and $T: \Omega_{q} \longrightarrow \Omega_{N}, G \in C^{1}\left(\Omega_{N}\right)$ and $T \in C^{1}\left(\Omega_{q}\right)$, such that the map $H$ of Hypothesis 2.1 can be expressed as

$$
H=T \circ G \text {. }
$$

The reduction procedure is carried out in two steps. The first one uses Hypothesis 2.1 to obtain the so-called auxiliary system:

$$
X_{n+1}=H\left(X_{n}\right)
$$


which is still a system of the same dimension $N$ as the original system (2.1). Now, using Hypothesis 2.2 we define the global variables by

$$
Y_{n}:=G\left(X_{n}\right) \in \mathbb{R}^{q} .
$$

The approximated reduced system, aggregated system, that we propose, represents the dynamics of these global variables in the auxiliary system (2.2):

$$
Y_{n+1}=G\left(X_{n+1}\right)=G\left(H\left(X_{n}\right)\right)=G\left(T\left(G\left(X_{n}\right)\right)\right)=G\left(T\left(Y_{n}\right)\right) .
$$

Defining $\bar{H}:=G \circ T$, the aggregated system reads as follows:

$$
Y_{n+1}=\bar{H}\left(Y_{n}\right)
$$

The relationship between the auxiliary and the aggregated systems is an example of perfect aggregation in the sense of [16]. To be precise, if $\left\{X_{n}\right\}_{n \in \mathbb{N}}$ is the solution of the auxiliary system (2.2) associated to the initial condition $X_{0}$ and $\left\{Y_{n}\right\}_{n \in \mathbb{N}}$ is the solution of the aggregated system (2.3) for $Y_{0}=G\left(X_{0}\right)$, then they verify $X_{n}=T\left(Y_{n-1}\right)$ and $Y_{n}=G\left(X_{n}\right)$ for $n=1,2, \ldots$.

Once we have proposed a reduced system we establish conditions that allow us to study the asymptotic behavior of the original system (2.1) in terms of the behavior of the aggregated system (2.3). We distinguish the linear and the nonlinear cases. In the linear case, we will see that the elements deciding the asymptotic behavior of system (2.1) are well approximated by those of system (2.3). In the nonlinear case, approximation results are found for equilibria and periodic solutions.

\subsection{Linear Systems.}

In this section we assume that maps $\left\{H_{k}\right\}_{k \in \mathbb{N}}$ are linear. Since our aim is to apply the reduction procedure to population dynamics models, we assume that the matrices defining the maps $H_{k}$, that we also denote $H_{k}$, are nonnegative.

The original system (2.1) in the linear case has the form:

$$
X_{k, n+1}=H_{k} X_{k, n}
$$

where $H_{k} \in \mathbb{R}_{+}^{N \times N}$ for $k \in \mathbb{N}$.

We now translate Hypotheses 2.1 and 2.2 to the linear case:

Hypothesis 2.3. There exists a matrix $H$ which is the limit of $H_{k}$

$$
\lim _{k \longrightarrow \infty} H_{k}=H .
$$

The auxiliary system associated to system (2.4) is $X_{n+1}=H X_{n}$.

Let us now make use of the concept of allowability of a nonnegative matrix [34]. A nonnegative matrix is called row-allowable (column-allowable) if it has, at least, one positive entry in each row (column).

Hypothesis 2.4. There exist $q \in \mathbb{N}, q<N$, and two nonnegative matrices $G \in \mathbb{R}_{+}^{q \times N}$ column-allowable and $T \in \mathbb{R}_{+}^{N \times q}$ row-allowable such that $H$ can be expressed as their product:

$$
H=T G .
$$

In what follows we assume that Hypotheses 2.3 and 2.4 are met.

The aggregated system associated to the linear system (2.4) is the following linear system

$$
Y_{n+1}=\bar{H} Y_{n}
$$

where its associated matrix is

$$
\bar{H}:=G T \text {. }
$$


To compare the asymptotic behavior of the original and the aggregated systems we assume that matrix $\bar{H}$ is primitive. This implies, via the Perron-Frobenius Theorem [34], that $\bar{H}$ has a strictly dominant eigenvalue $\lambda_{\bar{H}}>0$, which moreover has a corresponding positive right column eigenvector $\mathbf{v}_{\bar{H}}$ and a positive left row eigenvector $\mathbf{u}_{\bar{H}}^{\top}$, where $\mathrm{T}$ denotes transposition. These eigen-elements, to which we refer as asymptotic eigen-elements, characterize the asymptotic behavior of the linear system (2.5): indeed, for any initial condition $Y_{0}$ the associated solution $\left\{Y_{n}\right\}_{n \in \mathbb{N}}$ verifies

$$
\lim _{n \rightarrow \infty} \frac{1}{\lambda_{\bar{H}}^{n}} Y_{n}=\frac{\mathbf{u}_{\bar{H}}^{\top} Y_{0}}{\mathbf{u}_{\bar{H}}^{\top} \mathbf{v}_{\bar{H}}} \mathbf{v}_{\bar{H}} .
$$

In the following theorem we establish how the asymptotic eigen-elements of the original system can be approximated through those of the aggregated system.

Theorem 2.5. Suppose that Hypotheses 2.3 and 2.4 hold and that matrix $\bar{H}$ of the aggregated system (2.5) is primitive. Let $\lambda_{\bar{H}}$ be its strictly dominant eigenvalue, and $\mathbf{v}_{\bar{H}}$ and $\mathbf{u}_{\bar{H}}^{\top}$ corresponding positive right column and left row eigenvectors. Then we can conclude that, for $k$ large enough, the matrix $H_{k}$ of the original system (2.4) is primitive and has a strictly dominant eigenvalue $\lambda_{H_{k}}$ and corresponding positive right column and left row eigenvectors $\mathbf{v}_{H_{k}}$ and $\mathbf{u}_{H_{k}}^{\top}$. Moreover, for any consistent norm $\|\cdot\|$ and for $k \rightarrow \infty$ we have:

1. $\lambda_{H_{k}}=\lambda_{\bar{H}}+\frac{\mathbf{u}_{\bar{H}}^{\top} G\left(H_{k}-H\right) T \mathbf{v}_{\bar{H}}}{\lambda_{\bar{H}} \mathbf{u}_{\bar{H}}^{\top} \mathbf{v}_{\bar{H}}}+O\left(\left\|H_{k}-H\right\|^{2}\right)$.

2. $\mathbf{v}_{H_{k}}=T \mathbf{v}_{\bar{H}}+O\left(\left\|H_{k}-H\right\|\right)$.

3. $\mathbf{u}_{H_{k}}^{\top}=\mathbf{u}_{\bar{H}}^{\top} G+O\left(\left\|H_{k}-H\right\|\right)$.

Proof. The asymptotic eigen-elements of matrix $H$ associated to the auxiliary system are $\lambda_{\bar{H}}, T \mathbf{v}_{\bar{H}}$ and $\mathbf{u}_{\bar{H}}^{\top} G$. To obtain the expressions for the asymptotic eigen-elements of the original system we consider matrix $H_{k}$ as a perturbation of matrix $H$ and apply certain results on matrix perturbations [35]. A detailed proof can be followed in [26].

Summing up, we have shown that we can get essential information about the asymptotic behavior of the original system through the study of the aggregated system.

\subsection{Nonlinear Systems.}

In [33] the behavior of the solution of systems (2.1) and (2.3) are related for large enough values of parameter $k$, in the case $\left\{H_{k}\right\}_{k \in \mathbb{N}}$ is a sequence of nonlinear maps verifying Hypotheses 2.1 and 2.2. The results are of two kinds: on the one hand, [33] shows that, for a fixed value of $n$, the solutions of the system (2.1) converges to the corresponding solutions of system (2.3) when $k$ tends to infinity. On the other hand, [33] gives some asymptotic results concerning the existence and stability of fixed points and periodic solutions for both systems.

Here we focus our attention on the result ensuring the existence and stability of equilibria of the original system if this is the case for the aggregated system. Roughly speaking, it guarantees that under certain assumptions, the existence of an equilibrium $Y^{*}$ for the aggregated system implies, for large enough values of $k$, the existence of an equilibrium $X_{k}^{*}$ for the original system, which can be approximated in terms of $Y^{*}$. Moreover, if $Y^{*}$ is hyperbolic and asymptotically stable (unstable) so is $X_{k}^{*}$ and the basin of attraction of the latter can be approximated in terms of the basin of attraction of the former.

In the next theorem we denote $D H_{k}$ and $D H$ the differentials of the maps $H_{k}$ and $H$ respectively.

Theorem 2.6. Let us assume that $H_{k}, H \in C^{1}\left(\Omega_{N}\right)$, verify Hypotheses 2.1 and 2.2, and that

$$
\lim _{k \longrightarrow \infty} H_{k}=H \text { and } \lim _{k \longrightarrow \infty} D H_{k}=D H
$$

uniformly on any compact set $K \subset \Omega_{N}$. 
Let $Y^{*} \in \mathbb{R}^{q}$ be a hyperbolic equilibrium point of (2.3). Then there exists $k_{0} \in \mathbb{N}$ such that for each $k \geq k_{0}$ there exists a hyperbolic equilibrium point $X_{k}^{*}$ of (2.1) satisfying

$$
\lim _{k \rightarrow \infty} X_{k}^{*}=X^{*}
$$

where $X^{*}=T\left(Y^{*}\right)$. Moreover,

1. If $Y^{*}$ is asymptotically stable then $X_{k}^{*}$ is asymptotically stable, for each $k \geq k_{0}$, and if $X_{0} \in \mathbb{R}^{N}$ is such that $\lim _{n \longrightarrow \infty} \bar{H}^{n}\left(Y_{0}\right)=Y^{*}$, where $Y_{0}=G\left(X_{0}\right)$, then

$$
\lim _{n \longrightarrow \infty} H_{k}^{n}\left(X_{0}\right)=X_{k}^{*}
$$

2. If $Y^{*}$ is unstable then $X_{k}^{*}$ is unstable, for each $k \geq k_{0}$.

An analogous result can be stated for periodic solutions, see [33].

Condition (2.6) is in general difficult to check in practical applications, particularly the uniform convergence of the differentials. In the applications developed in Section 4 we show some results enabling condition (2.6) to hold so that the reduction procedure and the results relating the behavior of the original and the aggregated system can be applied.

\section{Slow-fast discrete models of population dynamics.}

The main goal of this section consists of describing the application of the general reduction procedure described in Section 2 to a general class of population dynamics discrete models including two time scales. Details can be found in [33].

Let us suppose a population generally divided into groups, and each of these groups divided into several subgroups. We can think, for instance, of an age-structured population occupying a multi-patch environment. In this case the population can be considered divided into groups which are the age classes, and each group divided into subgroups which are the individuals of the age class inhabiting each of the different patches.

The state at time $n$ of a population with $q$ groups is represented by a vector $X_{n}:=\left(\mathbf{x}_{n}^{1}, \ldots, \mathbf{x}_{n}^{q}\right)^{\top} \in \mathbb{R}_{+}^{N}$, where every vector $\mathbf{x}_{n}^{i}:=\left(x_{n}^{i 1}, \ldots, x_{n}^{i N^{i}}\right)^{\top} \in \mathbb{R}_{+}^{N^{i}}, i=1, \ldots, q$, represents the state of the $i$ group which is divided into $N^{i}$ subgroups, with $N=N^{1}+\cdots+N^{q}$.

In the evolution of the population we will consider two processes. We suppose that the characteristic time scales of both processes are very different from each other and so we will refer to them as the fast and slow processes or, still, as the fast and slow dynamics, which are defined respectively by two maps

$$
F, S: \Omega_{N} \longrightarrow \Omega_{N} \quad ; \quad F, S \in C^{1}\left(\Omega_{N}\right)
$$

where $\Omega_{N} \subset \mathbb{R}^{N}$ is a nonempty open set.

In order to write a single discrete model combining both processes, and therefore their different time scales, we have to choose its time unit. Two possible and reasonable choices are the time units associated to each one of the two processes. Here we use as the time unit of the model that corresponding to the slow dynamics $(S)$. We then need to approximate the effect of the fast dynamics $(F)$ over a time interval much longer than its own. To do so we will suppose that during each projection interval corresponding to the slow process map $F$ has operated a number $k$ of times, where $k$ is a big enough integer that can be interpreted as the ratio between the projection intervals corresponding to the slow and fast dynamics. Therefore, the fast dynamics will be modeled by $F^{k}$, the $k$-th iterate of $F$, and the complete system is defined by

$$
X_{k, n+1}=S\left(F^{k}\left(X_{k, n}\right)\right)
$$

where $X_{k, n} \in \mathbb{R}^{N}$ is the vector of state variables at time $n$. 
Notice that the setting of Section 2 corresponds to taking

$$
H_{k}:=S \circ F^{k},
$$

and then, in order to meet Hypotheses 2.1 and 2.2 we assume the following two hypotheses on $F$ :

Hypothesis 3.1. The sequence of iterates of $F,\left\{F^{k}\right\}_{k \in \mathbb{N}}$, converges pointwise on $\Omega_{N}$ to a map $\bar{F}$ : $\Omega_{N} \rightarrow \Omega_{N}$, such that $\bar{F} \in C^{1}\left(\Omega_{N}\right)$.

Hypothesis 3.2. There exist a non-empty open subset $\Omega_{q} \subset \mathbb{R}^{q}$ with $q<N$ and two maps $G: \Omega_{N} \longrightarrow$ $\Omega_{q}$ and $E: \Omega_{q} \longrightarrow \Omega_{N}$ with $G \in C^{1}\left(\Omega_{N}\right), E \in C^{1}\left(\Omega_{q}\right)$, such that the map $\bar{F}$ of Hypothesis 3.1 can be expressed as $\bar{F}=E \circ G$.

Now, as in Section 2, we proceed to reduce system (3.1) in two steps. First, we define the auxiliary system which approximates (3.1) when $k \rightarrow \infty$, i.e., when the fast process has reached an equilibrium. Denoting its vector state at time $n$ by $X_{n}$, this auxiliary system is

$$
X_{n+1}=S\left(\bar{F}\left(X_{n}\right)\right)=(S \circ E \circ G)\left(X_{N}\right) .
$$

Second, we define the global variables through

$$
Y_{n}:=G\left(X_{n}\right) \in \mathbb{R}^{q} .
$$

Applying $G$ to both sides of (3.2) we obtain the aggregated system associated to system (3.1)

$$
Y_{n+1}=(G \circ S \circ E)\left(Y_{n}\right) .
$$

Recall that the setting of Section 2 corresponds to taking $H:=S \circ \bar{F}=S \circ E \circ G$ and $T:=S \circ E$. Therefore, the aggregated system is associated to the map $\bar{H}:=G \circ S \circ E$.

In linear models of population dynamics, the linear maps $F$ and $S$ are defined by nonnegative matrices and then, assuming that the corresponding matrix $\bar{H}$ is primitive, Theorem 2.5 applies.

In nonlinear models we focus our attention on relating the asymptotic behavior of systems (3.1) and (3.3) for big enough values of parameter $k$. Then, in order to guarantee that the hypotheses of Theorem 2.6 hold, we require the following assumption on $F$ :

Hypothesis 3.3. The mappings $F$ and $\bar{F}$ satisfy that:

$$
\text { (i) } \lim _{k \rightarrow \infty} F^{k}=\bar{F} \quad, \quad \text { (ii) } \lim _{k \rightarrow \infty} D F^{k}=D \bar{F}
$$

uniformly on any compact set $K \subset \Omega_{N}$.

\section{Applications.}

\subsection{Linear Systems.}

We illustrate in this section the reduction of a linear discrete model with two time scales. We propose a simple population model coupling slow demography with fast migrations. Concerning demography, we consider the basic life cycle division ([24]), the population is structured into non-reproducing juveniles and reproducing adults. On the other hand, individuals in the population move between two different patches.

We represent the state of the population at time $n$ by vector

$$
X_{n}:=\left(x_{n}^{11}, x_{n}^{12}, x_{n}^{21}, x_{n}^{22}\right)^{\top} \in \mathbb{R}_{+}^{4},
$$


where $x^{1 j}$ and $x^{2 j}$ denote respectively the density of juveniles and adults in patch $j$, with $j=1,2$.

The matrix $F$ representing migrations is formed by two blocks $F_{i}, i=1,2$, corresponding to juveniles and adults. Each block is a simple regular stochastic matrix,

$$
F_{i}=\left(\begin{array}{cc}
1-q_{i} & p_{i} \\
q_{i} & 1-p_{i}
\end{array}\right) \quad \text { and } \quad F=\operatorname{diag}\left(F_{1}, F_{2}\right),
$$

where $q_{i}, p_{i} \in(0,1)$ are the proportions of individuals leaving respectively patches 1 and 2 .

The demography is defined by the survival rates of juveniles and adults and the fertility rate of adults. Juveniles mature in a single period of time. For each patch $j$ let $\sigma_{1 j}$ and $\sigma_{2 j}$ be the fraction of the juveniles and adults alive at time $n$ that survive to time $n+1$. Assuming that reproduction happens at the end of each period of time $[n, n+1)$, let $\phi_{j}$ be the number of juveniles produced by an adult that survive to time $n+1$ in patch $j$. The demography projection matrix thus reads

$$
S=\left(\begin{array}{cccc}
0 & 0 & \phi_{1} \sigma_{21} & 0 \\
0 & 0 & 0 & \phi_{2} \sigma_{22} \\
\sigma_{11} & 0 & \sigma_{21} & 0 \\
0 & \sigma_{12} & 0 & \sigma_{22}
\end{array}\right)
$$

We now put together slow demography and fast migrations, assuming that during each projection interval corresponding to demography, map F, which is associated to migrations, acts a number $k$ of times. The complete model that we propose is

$$
X_{k, n+1}=S F^{k} X_{k, n} .
$$

The fact that matrices $F_{i}$ are stochastic and primitive implies that Hypothesis 3.1 is verified

$$
\bar{F}=\lim _{k \rightarrow \infty} F^{k}=\lim _{k \rightarrow \infty} \operatorname{diag}\left(F_{1}^{k}, F_{2}^{k}\right)=\operatorname{diag}\left(\bar{F}_{1}, \bar{F}_{2}\right)
$$

with

$$
\bar{F}_{i}:=\left(\begin{array}{ll}
\frac{p_{i}}{p_{i}+q_{i}} & \frac{p_{i}}{p_{i}+q_{i}} \\
\frac{q_{i}}{p_{i}+q_{i}} & \frac{q_{i}}{p_{i}+q_{i}}
\end{array}\right) \quad, \quad i=1,2
$$

and also Hypothesis 3.2:

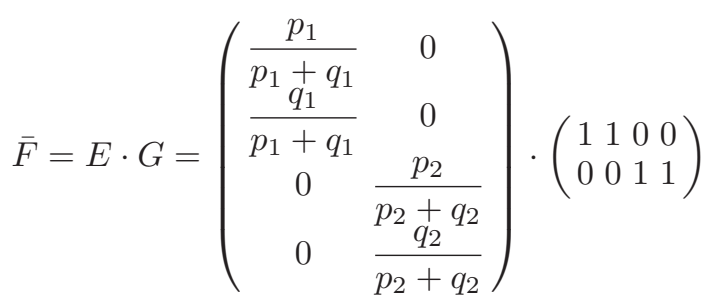

so that the global variables:

$$
Y=\left(\begin{array}{l}
y^{1} \\
y^{2}
\end{array}\right)=G X=\left(\begin{array}{l}
x^{11}+x^{12} \\
x^{21}+x^{22}
\end{array}\right)
$$

are the total densities of juveniles and adults, respectively, and the resulting aggregated system is

$$
Y_{n+1}=(G \cdot S \cdot E) Y_{n}=\left(\begin{array}{cc}
0 & \frac{\phi_{1} \sigma_{21} p_{2}+\phi_{2} \sigma_{21} q_{2}}{p_{2}+q_{2}} \\
\frac{\sigma_{11} p_{1}+\sigma_{12} q_{1}}{p_{1}+q_{1}} & \frac{\sigma_{21} p_{2}+\sigma_{21} q_{2}}{p_{2}+q_{2}}
\end{array}\right) Y_{n}
$$

Now, if the matrix $\bar{H}:=G \cdot S \cdot E$ is primitive, Theorem 2.5 applies and the dominant eigen-elements of system (4.3) can be approximated from the corresponding eigen-elements of system (4.4) with a discrepancy 
which is $O(1 / k)$. In particular this allows us to express conditions for the extinction or non-extinction of the population in a simple closed form. We consider $R$, the inherent net reproductive number of a matrix [12], that has the property of being larger, equal or less than 1 if and only if the dominant eigenvalue of the matrix is larger, equal or less than 1. For matrix $\bar{H}$ we have

$$
R=\frac{\left(\sigma_{11} p_{1}+\sigma_{12} q_{1}\right)\left(\phi_{1} \sigma_{21} p_{2}+\phi_{2} \sigma_{21} q_{2}\right)}{\left(p_{2}\left(1-\sigma_{21}\right)+q_{2}\left(1-\sigma_{22}\right)\right)\left(p_{1}+q_{1}\right)}
$$

and so we obtain ([12], Th. 1.1.3) that the solutions to the aggregated system grow exponentially if $R>1$ and decay exponentially if $R<1$. Via Theorem 2.5 we get, for $k$ large enough, the same conclusions on the solutions to the complete system (4.3). Though we presented a model with two patches, the procedure and the results would still hold in a similar model with an arbitrary number of patches.

Let us now propose a more realistic version of the preceding model. For species with separate generations, reproduction and maturation can be taken as processes happening once at the end of each period of time $[n, n+1)$, whereas mortality could happen at any time between $n$ and $n+1$. This means that mortality and survival should rather act at the fast time scale as migrations do. To take this fact into account while keeping the rest of assumptions included in model (4.3), we express the matrix $S$ (4.2) in the following form

$$
S_{k}=\left(\begin{array}{cccc}
0 & 0 & \phi_{1} & 0 \\
0 & 0 & 0 & \phi_{2} \\
1 & 0 & 1 & 0 \\
0 & 1 & 0 & 1
\end{array}\right) \cdot\left(\begin{array}{cccc}
\sigma_{11}^{1 / k} & 0 & 0 & 0 \\
0 & \sigma_{12}^{1 / k} & 0 & 0 \\
0 & 0 & \sigma_{21}^{1 / k} & 0 \\
0 & 0 & 0 & \sigma_{22}^{1 / k}
\end{array}\right)^{k}:=\bar{S} \cdot\left(\Sigma_{k}\right)^{k}
$$

and combine it with matrix $F(4.1)$ to propose the new complete model

$$
X_{k, n+1}=\bar{S}\left(\Sigma_{k} F\right)^{k} X_{k, n} .
$$

This system has a slightly more general form than (3.1) but denoting $H_{k}:=\bar{S}\left(\Sigma_{k} F\right)^{k}$ we have a particular case of system (2.1). We will now prove that system (4.5) verifies Hypotheses 2.3 and 2.4 so that Theorem 2.5 applies.

First of all, we need to find $H:=\lim _{k \rightarrow \infty} H_{k}$. In [25] the following result on the convergence of a matrix sequence is proved.

Theorem 4.1. Let $M$ be a primitive nonnegative matrix of order $N$ such that 1 is its strictly dominant eigenvalue. Let $\mathbf{v}$ and $\mathbf{u}^{\top}$ be its corresponding positive right column and left row eigenvectors chosen so that $\mathbf{u}^{\top} \mathbf{v}=1$. Let $D$ be a nonsingular matrix of order $N$ and $D^{*}$ a matrix such that $\exp \left(D^{*}\right)=D$, and let define matrices $D_{k}=\exp \left(\frac{1}{k} D^{*}\right)$. Then

$$
\lim _{k \rightarrow \infty}\left(D_{k} M\right)^{k}=\lim _{k \rightarrow \infty}\left(M D_{k}\right)^{k}=\gamma \mathbf{v} \mathbf{u}^{\top},
$$

where $\gamma=\exp \left(\mathbf{u}^{\top} D^{*} \mathbf{v}\right)$, and, furthermore, $\left\|\left(M D_{k}\right)^{k}-\gamma \mathbf{v} \mathbf{u}^{\top}\right\|=O(1 / k)$.

The matrix $\Sigma_{k} F$ is composed of two blocks of the form required to apply Theorem 4.1. Denoting

$$
D_{i, k}:=\left(\begin{array}{cc}
\left(\sigma_{i 1}\right)^{\frac{1}{k}} & 0 \\
0 & \left(\sigma_{i 2}\right)^{\frac{1}{k}}
\end{array}\right) \text { and } M_{i}:=\left(\begin{array}{cc}
1-q_{i} & p_{i} \\
q_{i} & 1-p_{i}
\end{array}\right) \quad, \quad i=1,2
$$

we have

$$
\Sigma_{k} F=\operatorname{diag}\left(D_{1, k} M_{1}, D_{2, k} M_{2}\right)
$$

Following the notation in Theorem 4.1 we denote $D_{i}:=\operatorname{diag}\left(\sigma_{i 1}, \sigma_{i 2}\right)$ and $D_{i}^{*}:=\operatorname{diag}\left(\ln \left(\sigma_{i 1}\right), \ln \left(\sigma_{i 2}\right)\right)$ and so we have $D_{i, k}=\exp \left(\frac{1}{k} D_{i}^{*}\right)$. Bearing in mind that $p_{i}, q_{i} \in(0,1)$, matrices $M_{i}$ are stochastic 
and primitive, so we can choose the eigenvectors associated to eigenvalue 1 to be $\mathbf{u}_{i}^{\top}=(1,1)$ and $\mathbf{v}_{i}=\left(\frac{p_{i}}{p_{i}+q_{i}}, \frac{q_{i}}{p_{i}+q_{i}}\right)^{\top}$. Calculating

$$
\gamma_{i}=\exp \left(\mathbf{u}_{i}^{\top} D_{i}^{*} \mathbf{v}_{i}\right)=\left(\sigma_{i 1}\right)^{\frac{p_{i}}{p_{i}+q_{i}}}\left(\sigma_{i 2}\right)^{\frac{q_{i}}{p_{i}+q_{i}}}
$$

we obtain from Theorem 4.1 the following limit

$$
\lim _{k \rightarrow \infty} D_{i, k} M_{i}=\gamma_{i} \mathbf{v}_{i} \mathbf{u}_{i}^{\top}
$$

and, therefore,

$$
H=\lim _{k \rightarrow \infty} H_{k}=\lim _{k \rightarrow \infty} \bar{S}\left(\Sigma_{k} F\right)^{k}=\bar{S} \operatorname{diag}\left(\gamma_{1} \mathbf{v}_{1} \mathbf{u}_{1}^{\top}, \gamma_{2} \mathbf{v}_{2} \mathbf{u}_{2}^{\top}\right)
$$

which implies that Hypothesis 2.3 holds for system (4.5).

Now we denote $G:=\operatorname{diag}\left(\mathbf{u}_{1}^{\top}, \mathbf{u}_{2}^{\top}\right)$ and $T:=\bar{S} \operatorname{diag}\left(\gamma_{1} \mathbf{v}_{1}, \gamma_{2} \mathbf{v}_{2}\right)$ and we have from (4.6) that $H=T \cdot G$, so Hypothesis 2.4 also holds for system (4.5).

The resulting aggregated system, for the global variables $Y=G X$, is

$$
Y_{n+1}=G T Y_{n}=\left(\begin{array}{cc}
0 & \gamma_{2} \frac{p_{2} \phi_{1}+q_{2} \phi_{2}}{p_{2}+q_{2}} \\
\gamma_{1} & \gamma_{2}
\end{array}\right) Y_{n}
$$

If we calculate the inherent net reproductive number of the matrix in (4.7)

$$
R=\frac{\gamma_{1} \gamma_{2}}{1-\gamma_{2}} \cdot \frac{p_{2} \phi_{1}+q_{2} \phi_{2}}{p_{2}+q_{2}}
$$

we obtain from theorem 2.5 that, for $k$ large enough, solutions of system (4.5) grow exponentially if $R>1$ and decay exponentially if $R<1$.

\subsection{Nonlinear Systems I: Fast dynamics defined by a matrix depending on global groups densities.}

In this section we illustrate the theoretical results established in Section 3 by means of some applications. The main difficulty consists of proving that Hypothesis 3.3 is met. Here we present a class of models for which this holds and so Theorem 2.6 applies.

Following [27], we will suppose that for each group $i=1, \ldots, q$, the fast dynamics is internal, conservative of the total number of individuals and with an asymptotically stable distribution among the groups. These assumptions are met in the particular case of representing the fast dynamics for each group $i$ by a projection matrix which is a regular stochastic matrix of dimensions $N^{i} \times N^{i}$. Hypothesis 3.3 is trivially satisfied if these projection matrices are constant. In what follows we will extend this situation to the nonlinear case in which such projection matrices depend on the total number of individuals in each group.

To start with, let us introduce some definitions. Let us set $\mathbf{1}_{i}:=(1, \ldots, 1)^{\top} \in \mathbb{R}^{N_{i}}, i=1, \ldots, q$, $\mathcal{U}:=\operatorname{diag}\left(\mathbf{1}_{1}^{\top}, \ldots, \mathbf{1}_{q}^{\top}\right)$ and $\Omega_{q}:=\mathcal{U} \Omega_{N} \subset \mathbb{R}^{q}$.

For each $i=1, \ldots, q$, let $P_{i}(\cdot) \in C^{1}\left(\Omega_{q}\right)$ be a matrix function such that for each $Y \in \Omega_{q}, P_{i}(Y)$ is a regular stochastic matrix of dimensions $N^{i} \times N^{i}$. As a consequence, 1 is a simple and strictly dominant in modulus eigenvalue for $P_{i}(Y)$, with associated right and left eigenvectors $\mathbf{v}_{i}(Y), \mathbf{1}_{i}$ respectively. The eigenvector $\mathbf{v}_{i}(Y)$ can be chosen as the asymptotically stable probability distribution, i.e., $\mathbf{v}_{i}(Y) \geq 0$ and $\mathbf{1}_{i}^{\top} \mathbf{v}_{i}(Y)=1, i=1, \ldots, q$.

The fast dynamics for the whole population is then represented by the block diagonal matrix

$$
\forall Y \in \Omega_{q} \quad, \quad \mathcal{F}(Y):=\operatorname{diag}\left(P_{1}(Y), \ldots, P_{q}(Y)\right) .
$$


The Perron-Frobenius Theorem applies to each matrix $P_{i}(Y)$, so that we have

$$
\bar{P}_{i}:=\lim _{k \rightarrow \infty} P_{i}^{k}(Y)=\left(\mathbf{v}_{i}(Y)|\ldots| \mathbf{v}_{i}(Y)\right)=\mathbf{v}_{i}(Y) \mathbf{1}_{i}^{\top} .
$$

Introducing the notations

$$
\overline{\mathcal{F}}(Y):=\operatorname{diag}\left(\bar{P}_{1}(Y), \ldots, \bar{P}_{q}(Y)\right) \quad, \quad \mathcal{V}(Y):=\operatorname{diag}\left(\mathbf{v}_{1}(Y), \ldots, \mathbf{v}_{q}(Y)\right),
$$

we also have

$$
\forall Y \in \Omega_{q} \quad, \quad \overline{\mathcal{F}}(Y)=\lim _{k \rightarrow \infty} \mathcal{F}^{k}(Y)=\mathcal{V}(Y) \mathcal{U} .
$$

Finally, the nonlinear model that we are considering is formulated as

$$
X_{k, n+1}=S\left(\mathcal{F}^{k}\left(\mathcal{U} X_{k, n}\right) X_{k, n}\right),
$$

where $S \in C^{1}\left(\Omega_{N}\right)$ stands for the slow process, as in Section 3.

If we think that the ratio of slow to fast time scales tends to infinity, i.e. $k \rightarrow \infty$, or in other words, that the fast process is instantaneous in relation to the slow one, proceeding as in Section 3 we can approximate system (4.8) by the auxiliary system

$$
X_{n+1}=S\left(\overline{\mathcal{F}}\left(\mathcal{U} X_{n}\right) X_{n}\right)=S\left(\mathcal{V}\left(\mathcal{U} X_{n}\right) \mathcal{U} X_{n}\right) .
$$

We see that the evolution of this system depends on $\mathcal{U} X_{n} \in \mathbb{R}^{q}$, which suggests the global variables should be defined by

$$
Y_{n}:=\mathcal{U} X_{n}
$$

and therefore the aggregated system of system (4.8) is

$$
Y_{n+1}=\mathcal{U} S\left(\mathcal{V}\left(Y_{n}\right) Y_{n}\right)
$$

Our main goal consists of establishing an approximation result between the solutions to the complete system (4.8) and the aggregated model (4.9). To this end, we will formulate both systems in the setting of Theorem 2.6.

The model (4.8) fits in the formulation given by (3.1) if we choose

$$
\forall X \in \Omega_{N} \quad, \quad F(X):=\mathcal{F}(\mathcal{U} X) X .
$$

Therefore, bearing in mind that for all $Y \in \mathbb{R}^{q}$ we have $\mathcal{U F}(Y)=\mathcal{U}$, we have that for all $X \in \Omega_{N}$

$$
\lim _{k \rightarrow \infty} F^{k}(X)=\lim _{k \rightarrow \infty} \mathcal{F}^{k}(\mathcal{U} X) X=\overline{\mathcal{F}}(\mathcal{U} X) X=\mathcal{V}(\mathcal{U} X) \mathcal{U} X
$$

and then

$$
\forall X \in \Omega_{N} \quad, \quad \bar{F}(X):=\mathcal{V}(\mathcal{U} X) \mathcal{U} X .
$$

Notice that functions $G$ and $E$ mentioned in Hypothesis 3.2 correspond to taking $G(X):=\mathcal{U} X$ and $E(Y):=\mathcal{V}(Y) Y$.

To apply Theorem 2.6 we have to check that Hypotheses 3.1, 3.2 and 3.3 are satisfied in this formulation. We only provide an outline of the proof, the technical details being explained in [22].

The regularity imposed in Hypotheses 3.1 and 3.2 hold immediately from the $C^{1}$ regularity of eigenvectors $\mathbf{v}_{i}(\cdot), i=1, \ldots, q$, as established in [22]. Regarding Hypothesis 3.3, notice that for each $Y \in \Omega_{q}$, matrix $\mathcal{F}(Y)$ can be written as

$$
\mathcal{F}(Y)=(\mathcal{V}(Y) \mid R(Y))\left(\begin{array}{cc}
I_{q} & O \\
O & J(Y)
\end{array}\right)\left(\begin{array}{c}
\mathcal{U} \\
T(Y)
\end{array}\right)=\mathcal{V}(Y) \mathcal{U}+Q(Y)
$$


where $Q(Y):=R(Y) J(Y) T(Y)$, being $R(Y)$ and $T(Y)$ suitable matrices and $J(Y)$ corresponding to the Jordan blocks of $\mathcal{F}(Y)$ associated to eigenvalues of modulus strictly less than 1. Therefore, for all $Y \in \Omega_{q}$, the spectral radius of $Q(Y)$ is strictly less than 1. Moreover, straightforward calculations lead to

$$
\mathcal{F}^{k}(Y)=\mathcal{V}(Y) \mathcal{U}+Q^{k}(Y) \quad, \quad k=1,2, \ldots
$$

and then, since $\mathcal{V}, \mathcal{F} \in C^{1}\left(\Omega_{q}\right)$, we also have $Q \in C^{1}\left(\Omega_{q}\right)$.

This regularity of $Q$ implies the continuity of the spectral radius $\rho(Q(\cdot))$ in $\Omega_{q}$, which in turn allows us to assure that, for each compact set $K_{q} \subset \Omega_{q}$, we have

$$
\sup _{Y \in K_{q}}\left\|Q^{k}(Y)\right\| \rightarrow 0(k \rightarrow \infty)
$$

Now, since

$$
\left\|F^{k}(\mathcal{U} X)-\bar{F}(X)\right\|=\left\|\mathcal{F}^{k}(\mathcal{U} X) X-\mathcal{V}(\mathcal{U} X) \mathcal{U} X\right\| \leq\left\|Q^{k}(\mathcal{U} X)\right\|\|X\|
$$

condition (i) in Hypothesis 3.3 holds.

On the other hand, notice that (4.10) implies that

$$
\forall X \in \Omega_{N} \quad, \quad D F^{k}(X)=D \bar{F}(X)+D\left[Q^{k}(\mathcal{U} X) X\right] .
$$

A technical proof whose details can be found in [22] shows that, for each compact set $K_{N} \subset \Omega_{N}$, we have

$$
\sup _{X \in K_{N}}\left\|D\left[Q^{k}(\mathcal{U} X) X\right]\right\| \rightarrow 0 \quad(k \rightarrow \infty)
$$

from which condition (ii) in Hypothesis 3.3 can be proved.

Summing up, Theorem 2.6 applies, providing the following approximation result:

Theorem 4.2. Let $Y^{*} \in \mathbb{R}^{q}$ be a hyperbolic equilibrium of system (4.9). Then, there exists an integer $k_{0} \geq 0$ such that for all $k \geq k_{0}$, system (4.8) has an equilibrium point $X_{k}^{*}$ which is hyperbolic and satisfies

$$
\lim _{k \rightarrow \infty} X_{k}^{*}=X^{*} \quad \text { where } \quad X^{*}:=S\left(\mathcal{V}\left(Y^{*}\right) Y^{*}\right)
$$

Moreover, the following holds:

(i) $X_{k}^{*}$ is asymptotically stable (resp. unstable) if and only if $Y^{*}$ is asymptotically stable (resp. unstable).

(ii) Let $Y^{*}$ be asymptotically stable and let $X_{0} \in \Omega_{N}$ be such that the solution $\left\{Y_{n}\right\}_{n=0,1, \ldots}$ to (4.9) corresponding to the initial data $Y_{0}:=\mathcal{U} X_{0}$ satisfies that $\lim _{n \rightarrow \infty} Y_{n}=Y^{*}$. Then, for all $k \geq k_{0}$, the solution to (4.8) $\left\{X_{k, n}\right\}_{n=0,1, \ldots}$ with $X_{k, 0}=X_{0}$ satisfies that $\lim _{n \rightarrow \infty} X_{k, n}=X_{k}^{*}$.

In some applications, it would be more realistic to have the fast dynamics dependent on the state variables, and not just on the global variables as in Theorem 4.2. Nevertheless, it does not seem easy to find a proof for this more general case and specific proofs should be provided for each particular case of fast dynamics depending on state variables. We deal with this case in Section 4.3.

\subsubsection{An example: Non-structured multi-patch models with fast migrations.}

In this section we will present some interesting applications within the framework of Theorem 4.2. Let us consider a non-structured population inhabiting a multi-patch environment, so that the corresponding aggregated model is a scalar nonlinear difference equation. That is, the population is constituted by just one group of individuals which is subdivided into subgroups representing the local populations at the patches making up its habitat.

For the sake of simplicity, we will consider a two-patch environment, and the local dynamics depending only on the local population. To be precise, the population vector at time $n$ is $X_{n}:=\left(x_{n}^{1}, x_{n}^{2}\right)^{\top}$ and the slow dynamics is described by

$$
S\left(X_{n}\right):=\left(s_{1}\left(x_{n}^{1}\right), s_{2}\left(x_{n}^{2}\right)\right)^{\top},
$$


where $s_{i}(\cdot), i=1,2$, are two non-negative $C^{1}$ functions defined on $\mathbb{R}_{+}$.

The fast dynamics, associated in this model to the migration process, is represented by a regular stochastic matrix whose entries depend on the total population $y:=x^{1}+x^{2}$

$$
\mathcal{F}(y):=\left(\begin{array}{cc}
1-q(y) & p(y) \\
q(y) & 1-p(y)
\end{array}\right) \quad p, q: \mathbb{R}_{+} \longrightarrow(0,1) \quad, \quad p, q \in C^{1}\left(\mathbb{R}_{+}\right) .
$$

Therefore

$$
\overline{\mathcal{F}}(y)=\lim _{k \rightarrow \infty} \mathcal{F}^{k}(y)=(\mathbf{v}(y) \mid \mathbf{v}(y)) \quad, \quad \mathbf{v}(y)=\left(\begin{array}{c}
\frac{p(y)}{p(y)+q(y)} \\
\frac{q(y)}{p(y)+q(y)}
\end{array}\right):=\left(\begin{array}{l}
v_{1}(y) \\
v_{2}(y)
\end{array}\right) .
$$

A straightforward application of the results established in Section 4.2 leads to the aggregated model

$$
y_{n+1}=s_{1}\left(v_{1}\left(y_{n}\right) y_{n}\right)+s_{2}\left(v_{2}\left(y_{n}\right) y_{n}\right) .
$$

To illustrate how this aggregation procedure provides an explanation of some classical mono-species discrete models, assume that a malthusian dynamics acts at each patch, i.e.,

$$
S\left(X_{n}\right):=\left(d_{1} x_{n}^{1}, d_{2} x_{n}^{2}\right)^{\top} \quad \text { together with } 0<d_{1}<1<d_{2}
$$

which means that patch 1 behaves as a sink and path 2 as a source. A simple calculation (see [22]) shows that certain kinds of density dependent migrations can assure the existence of a positive asymptotically stable equilibrium for the aggregated model. Moreover, it can be shown that, for suitable choices of functions $q(y), p(y)$, the aggregated model recovers some classical discrete models, like the so-called Beverton-Holt or Ricker models. Also, some examples of local nonlinear dynamics different from malthusian, coupled with nonlinear fast migrations, are analyzed with some detail in [22], which give rise to a variety of situations, among them Allee-type effect dynamics.

\subsubsection{An age-structured model with fast demography.}

In this section we develop the reduction of a model of an age-structured population living in a multipatch habitat with the special feature of considering demography fast in comparison with migration. This model extends slightly the framework presented in Section 4.2 and can be considered as a generalization to nonlinear models of some results established in [30].

To be precise, let us consider an age-structured population distributed between two spatial patches. We will distinguish two age classes: juvenile (class 1, non reproductive) and adult (class 2, reproductive), so that the state of the population at time $n$ is represented by a vector

$$
X_{n}:=\left(\mathbf{x}_{n}^{1}, \mathbf{x}_{n}^{2}\right)^{\top} \in \mathbb{R}_{+}^{4} \quad, \quad \mathbf{x}_{n}^{i}:=\left(x_{n}^{i 1}, x_{n}^{i 2}\right)^{\top}, i=1,2,
$$

where $x_{n}^{i j}$ stands for the number of individuals of age class $j$ inhabitant patch $i$.

The demography is a local process driven by a Leslie $C^{1}$ matrix function

$$
\forall y \in \mathbb{R}_{+} \quad, \quad L_{i}(y):=\left(\begin{array}{cc}
0 & f_{12}^{i}(y) \\
t_{21}^{i}(y) & t_{22}^{i}(y)
\end{array}\right), i=1,2
$$

where, as usual, $f_{12}^{i}(\cdot)$ stands for the fertility rate of the adults and $t_{2 j}^{i}(\cdot), j=1,2$, represent the survival rate of each age class. As a consequence we can find associate positive right and left eigenvectors $\mathbf{v}_{i}(y)$, $\mathbf{u}_{i}^{\top}(y)$, which are chosen normalized by the condition $\mathbf{u}_{i}^{\top}(y) \mathbf{v}_{i}(y)=1$.

Then, the fast dynamics for the whole population is represented by the block diagonal matrix

$$
\forall Y:=\left(\begin{array}{c}
y^{1} \\
y^{2}
\end{array}\right) \in \mathbb{R}_{+}^{2} \quad, \quad \mathcal{L}(Y):=\left(\begin{array}{cc}
L_{1}\left(y^{1}\right) & 0 \\
0 & L_{2}\left(y^{2}\right)
\end{array}\right) .
$$


The general Perron-Frobenius theory of non-negative matrices applies, so that there exists the limit

$$
\forall y \in \mathbb{R}_{+} \quad, \quad \bar{L}_{i}(y):=\lim _{k \rightarrow \infty} L_{i}^{k}(y)=\mathbf{v}_{i}(y) \mathbf{u}_{i}^{\top}(y), i=1,2
$$

and therefore

$$
\overline{\mathcal{L}}(Y):=\lim _{k \rightarrow \infty} \mathcal{L}^{k}(Y)=\left(\begin{array}{cc}
\bar{L}_{1}\left(y^{1}\right) & 0 \\
0 & \bar{L}_{2}\left(y^{2}\right)
\end{array}\right)=\mathcal{V}(Y) \mathcal{U}(Y)
$$

where, as in Section 4.2, we are denoting

$$
\mathcal{V}(Y):=\operatorname{diag}\left(\mathbf{v}_{1}\left(y^{1}\right), \mathbf{v}_{2}\left(y^{2}\right)\right) \quad, \quad \mathcal{U}(Y):=\operatorname{diag}\left(\mathbf{u}_{1}^{\top}\left(y_{1}\right), \mathbf{u}_{2}^{\top}\left(y_{2}\right)\right) .
$$

In addition to demography, we consider migrations between patches. To keep things simple we consider a linear process represented by a constant stochastic matrix

$$
M:=\left(\begin{array}{cccc}
1-q_{1} & 0 & q_{2} & 0 \\
0 & 1-p_{1} & 0 & p_{2} \\
q_{1} & 0 & 1-q_{2} & 0 \\
0 & p_{1} & 0 & 1-p_{2}
\end{array}\right) \quad, \quad p_{i}, q_{i} \in(0,1), i=1,2
$$

where $q_{i}$ (resp. $p_{i}$ ) stands for the fraction of juvenile (resp. adult) individuals which move from patch $i$.

In this model we are assuming that demography is much faster than migrations and spatially internal, that is, only dependent on the population on each patch.

The theory developed in Section 4.2 does not exactly match with the setting here, but it can be easily adapted: everything works if the fast dynamics is given by a non-negative $C^{1}$ matrix function whose strictly dominant in modulus eigenvalue is 1 and the corresponding associated normalized left eigenvector is constant. These assumptions are met in this model assuming that the rates $t_{21}^{i}(\cdot)$ are constant for $i=1,2$ and also that

$$
\forall y \in \mathbb{R}_{+} \quad, \quad t_{22}^{i}(y)+f_{12}^{i}(y) t_{21}^{i}=1, i=1,2 .
$$

Straightforward calculations show that

$$
\mathbf{u}_{i}^{\top}(y)=\left(1, \frac{1}{t_{21}^{i}(y)}\right) \quad, \quad i=1,2
$$

so that, under the above assumptions, $\mathcal{U}(\cdot)$ is a constant matrix and the global variables are defined by

$$
Y_{n}:=\mathcal{U} X_{n}=\left(\begin{array}{c}
x_{n}^{11}+\left(1 / t_{21}^{1}\right) x_{n}^{12} \\
x_{n}^{21}+\left(1 / t_{21}^{2}\right) x_{n}^{22}
\end{array}\right):=\left(\begin{array}{c}
y_{n}^{1} \\
y_{n}^{2}
\end{array}\right),
$$

which are biologically meaningful, as they are the population at each patch weighted by its reproductive values. Therefore it makes sense to consider the fertility rates of each reproductive class as a function of the global variables, which implies that the rates $t_{22}^{i}(\cdot), i=1,2$ are also dependent on the global variables because of relation (4.11).

Summing up, the slow-fast model that we are considering is

$$
X_{k, n+1}=M \mathcal{L}^{k}\left(\mathcal{U} X_{k, n}\right) X_{k, n}
$$

which, arguing as in Section 4.2, can be reduced to the following nonlinear aggregated model expressed in terms of the global variables

$$
Y_{n+1}=\mathcal{U} M \mathcal{V}\left(Y_{n}\right) Y_{n}
$$

Numerical experiments carried out in [22] using a large range of values for the parameters illustrate the asymptotic behavior of the aggregated model, showing in particular that there are several scenarios for which there exists a unique positive asymptotically stable fixed point, as well as several scenarios 
for which there exist two different positive asymptotically stable fixed points. That is, multi-attractor scenarios are allowed.

Notice that, as the general slow-fast spatially structured models miss their spatial features when aggregation is performed, the reduction method developed in Section 4.2 allows one to study spatially distributed populations by means of reduced non-spatial models.

\subsection{Nonlinear Systems II: Fast dynamics defined by a matrix depending on subgroups densities.}

In this section we consider a host-parasitoid system in a spatial environment which is a chain of $m$ patches and in which the dispersal of host and parasitoids is fast with respect to the local host-parasitoid interactions in each patch. This model is based upon the one considered in [25].

Let $h_{n}^{i}$ and $p_{n}^{i}$ be, respectively, the host and parasitoid density on patch $i, i=1, \ldots, m$, at each generation $n$. We define the vectors $H_{n}:=\left(h_{n}^{1}, \ldots, h_{n}^{m}\right)^{\top}, P_{n}:=\left(p_{n}^{1}, \ldots, p_{n}^{m}\right)^{\top}$ corresponding to the population of hosts and parasitoids respectively. Therefore, the population vector is given by

$$
X_{n}:=\left(h_{n}^{1}, \ldots, h_{n}^{m}, p_{n}^{1}, \ldots, p_{n}^{m}\right)^{\top}=\left(\begin{array}{c}
H_{n} \\
P_{n}
\end{array}\right) \in \mathbb{R}^{2 m}
$$

Using the terminology of Section 3, the groups correspond to the division of the population in hosts and parasitoids, and the subgroups correspond to the distribution of hosts and parasitoids in the different patches.

Let us consider the host-parasitoid dispersal submodel. We assume that individuals can move over a distance of $k$ patches at each generation in a diffusion-like dispersal process. In order to compute the distribution obtained for hosts and parasitoids, we discretize this process in time and decompose it into $k$ elementary dispersal events consisting of movements from one patch to one of its nearest neighbors. We suppose that hosts move according to an asymmetrical dispersal process, a biased random walk, while parasitoids dispersal is host density-dependent.

Host movements correspond to an asymmetric (biased) random walk: probabilities to go to the left or to the right patch are not the same. Let $f$ be the proportion of hosts moving from any patch to the neighboring patch situated on its left between two time steps of migration. We define parameter $\alpha>0$ and assume that the proportion of migrants from any patch to the neighboring patch on its right is $\alpha f$ at each elementary dispersal event. When $\alpha>1$, the proportion of hosts moving to the right is larger than in the opposite direction. Thus, after several elementary migration events, one expects to end up with a spatial host distribution shifted towards the right hand side of the chain. The contrary happens when $\alpha<1$. The proportion of hosts leaving a patch to go to left and right ones must be smaller than 1 , and thus we assume that $(1+\alpha) f<1$.

For parasitoids we assume a host density-dependent dispersal process. Dispersal corresponds to a random walk, but the probability of leaving the patch (and thus the proportion of migrants) depends on the density of hosts on that patch. If the parasitoid is unable to detect the presence of hosts thanks to the pheromones left by hosts, it will leave the patch. Let $g\left(h_{n}^{i}\right)$ be the proportion of parasitoids leaving any patch $i$ to go to the two neighboring patches at each elementary dispersal event, which only depends on the density of hosts on patch $i$ at time step $n$. In this work, $g\left(h_{n}^{i}\right)$ is given by a general classical type of function of the following form:

$$
g\left(h_{n}^{i}\right)=\frac{1}{1+\tau\left(h_{n}^{i}\right)^{\beta}}
$$

where $\tau$ and $\beta$ are positive parameters. A similar host density dependent dispersal process for parasitoids was used in the case of two spatial patches in [18]. Since changing parameter $\tau$ corresponds to changing the scale for hosts, we will assume that $\tau=1$ in all this study. 
We assume that parasitoids leaving patch $i$ move in equal proportions to the two neighboring patches. Then for any patch $1<i<m$, the elementary dispersal process reads as follows:

$$
\begin{aligned}
& h_{n+1}^{i}=[1-(1+\alpha) f] h_{n}^{i}+\alpha f h_{n}^{i-1}+f h_{n}^{i+1} \\
& p_{n+1}^{i}=\left[1-g\left(h_{n}^{i}\right)\right] p_{n}^{i}+\frac{1}{2} g\left(h_{n}^{i-1}\right) p_{n}^{i-1}+\frac{1}{2} g\left(h_{n}^{i+1}\right) p_{n}^{i+1}
\end{aligned}
$$

and for patches at both ends of the chain

$$
\begin{aligned}
h_{n+1}^{1} & =[1-\alpha f] h_{n}^{1}+f h_{n}^{2} \\
p_{n+1}^{1} & =\left[1-\frac{1}{2} g\left(h_{n}^{1}\right)\right] p_{n}^{1}+\frac{1}{2} g\left(h_{n}^{2}\right) p_{n}^{2} \\
h_{n+1}^{m} & =[1-f] h_{n}^{m}+\alpha f h_{n}^{m-1} \\
p_{n+1}^{m} & =\left[1-\frac{1}{2} g\left(h_{n}^{m}\right)\right] p_{n}^{m}+\frac{1}{2} g\left(h_{n}^{m-1}\right) p_{n}^{m-1}
\end{aligned}
$$

Therefore, assuming that the time unit $n \mapsto n+1$ corresponds to the fast dynamics, the elementary dispersal process can be described by system

$$
X_{n+1}=F\left(X_{n}\right)
$$

where function $F$ is given by

$$
F\left(X_{n}\right):=\mathcal{F}\left(X_{n}\right) X_{n}:=\left(\begin{array}{cc}
\mathcal{F}_{h} & 0 \\
0 & \mathcal{F}_{p}\left(H_{n}\right)
\end{array}\right)\left(\begin{array}{c}
H_{n} \\
P_{n}
\end{array}\right)
$$

where $\mathcal{F}_{h} \in \mathcal{M}_{m \times m}(\mathbb{R})$, which stands for host migrations, is given by

$$
\mathcal{F}_{h}=\left(\begin{array}{cccccc}
1-\alpha f & f & 0 & \cdots & \cdots & 0 \\
\alpha f & 1-(1+\alpha) f & f & \ddots & \cdots & 0 \\
0 & \ddots & \ddots & \ddots & \ddots & \vdots \\
\vdots & \ddots & \ddots & \ddots & \ddots & 0 \\
\vdots & \ddots & \ddots & \alpha f & 1-(1+\alpha) f & f \\
0 & \cdots & \cdots & 0 & \alpha f & 1-f
\end{array}\right)
$$

and $\mathcal{F}_{p}\left(H_{n}\right) \in \mathcal{M}_{m \times m}\left(\mathcal{C}^{1}(\mathbb{R})\right)$ defines parasitoid migrations, namely,

$$
\mathcal{F}_{p}\left(H_{n}\right)=\left(\begin{array}{cccccc}
1-\frac{g\left(h_{n}^{1}\right)}{2} & \frac{g\left(h_{n}^{2}\right)}{2} & 0 & \ldots & \ldots & 0 \\
\frac{g\left(h_{n}^{1}\right)}{2} & 1-g\left(h_{n}^{2}\right) & \frac{g\left(h_{n}^{3}\right)}{2} & \ddots & \ldots & 0 \\
0 & \ddots & \ddots & \ddots & \ddots & \vdots \\
\vdots & \ddots & \ddots & \ddots & \ddots & 0 \\
\vdots & \ddots & \ddots & \frac{g\left(h_{n}^{m-2}\right)}{2} & 1-g\left(h_{n}^{m-1}\right) & \frac{g\left(h_{n}^{m}\right)}{2} \\
0 & \ldots & \ldots & 0 & \frac{g\left(h_{n}^{m-1}\right)}{2} & 1-\frac{g\left(h_{n}^{m}\right)}{2}
\end{array}\right)
$$

Let us now consider the intergroups interactions, i.e, the host-parasitoid dynamics. We assume that on each patch $i=1, \ldots, m$ of the chain, the interaction between hosts and parasitoids takes place according to the Nicholson-Bailey model

$$
\left\{\begin{array}{l}
h_{n+1}^{i}=\lambda_{i} h_{n}^{i} e^{-a_{i} p_{n}^{i}}:=z_{i}\left(h_{n}^{i}, p_{n}^{i}\right) \\
p_{n+1}^{i}=c_{i} h_{n}^{i}\left(1-e^{-a_{i} p_{n}^{i}}\right):=k_{i}\left(h_{n}^{i}, p_{n}^{i}\right)
\end{array}\right.
$$


where $\lambda_{i}$ is the host growth rate on patch $i, a_{i}$ the searching efficiency of parasitoids and $c_{i}$ the average number of viable parasitoids that emerge from an host parasitized at the previous generation. Accordingly the slow dynamics is defined by the mapping $S: \mathbb{R}^{2 q} \rightarrow \mathbb{R}^{2 m}$ given by $S=\left(z_{1}, \ldots, z_{m}, k_{1}, \cdots, k_{m}\right)$, where functions $z_{i}$ and $k_{i}$, for $i=1, \ldots, m$, are defined by equation (4.16) and describe the local host-parasitoid interactions.

The complete model combines the two previous submodels. We consider the slow time scale as the time unit for this complete model: one time step corresponds to a single event of reproduction, and thus one generation. Therefore, it is assumed that between time $n$ and $n+1$ hosts and parasitoids first explore the environment by performing $k$ elementary dispersal events before settling down, then they enter a phase where they have local demographic and parasitism interactions. Therefore the complete model reads

$$
X_{k, n+1}=S \circ F^{k}\left(X_{k, n}\right)
$$

Now we will show that system (4.17) meets the three Hypotheses 3.1, 3.2 and 3.3 of Section 3 and, therefore it can be aggregated and certain important aspects of the dynamics of the complete model can be inferred from the dynamics of the reduced one.

The model we have proposed can be thought of as a particular case of a more general abstract setting that is studied in [23, Section 1.3]. Loosely speaking, in that work it is proved that approximate aggregation techniques work in an abstract framework in which the fast dynamics is conservative of the total number of individual and meets the following hypothesis: the groups can be split into two classes, those whose fast dynamics is linear (and therefore independent of the rest of the groups) and those whose fast dynamics depends only on the state of the groups on the first set. In our present setting, the first set of groups corresponds to the host population and the second group to the parasitoid population.

Following [23, Section 1.3], we will obtain the equilibrium dispersal distribution for the population. It is straightforward to note that the $k$-fold iteration of $F$ is given by

$$
F^{k}(H, P)=\left(\begin{array}{cc}
\mathcal{F}_{h}^{k} & 0 \\
0 & \mathcal{F}_{p}\left(\mathcal{F}_{h}^{k-1} H\right) \ldots \mathcal{F}_{p}\left(\mathcal{F}_{h}^{1} H\right) \mathcal{F}_{p}(H)
\end{array}\right)\left(\begin{array}{c}
H \\
P
\end{array}\right)
$$

The stable asymptotic distribution for the (linear) host migrations is given by the normalized right Perron eigenvector of $\mathcal{F}_{h}$, i.e.,

and therefore we can define

$$
\mathbf{v}_{h}:=\frac{1-\alpha}{1-\alpha^{m}}\left(1, \ldots, \alpha^{i-1}, \ldots, \alpha^{m-1}\right)^{\top}
$$

$$
\overline{\mathcal{F}}_{h}:=\lim _{k \rightarrow \infty} \mathcal{F}_{h}^{k}=\left(\mathbf{v}_{h}|\cdots| \mathbf{v}_{h}\right)=\mathbf{v}_{h} \mathbf{1}_{m}^{\top} .
$$

Regarding the dispersal equilibrium distribution of parasitoids, we will first compute this distribution assuming that the host population $H_{n}$ remains constant. The normalized right Perron eigenvector of $\mathcal{F}_{p}(H)$ is easily found to be

so that

$$
\mathbf{v}_{p}(H):=\frac{1}{m+\sum_{j=1}^{m} h_{j}^{\beta}}\left(1+h_{1}^{\beta}, \ldots, 1+h_{m}^{\beta}\right)^{\top}
$$

$$
\overline{\mathcal{F}}_{p}(H):=\lim _{k \rightarrow \infty} \mathcal{F}_{p}^{k}(H)=\left(\mathbf{v}_{p}(H)|\cdots| \mathbf{v}_{p}(H)\right)=\mathbf{v}_{p}(H) \mathbf{1}_{m}^{\top}
$$

The next result shows that Hypothesis 3.1 in Section 3 is met:

Proposition 4.3. $F^{k}$ converges pointwise in $\mathbb{R}_{+}^{2 m}$ to the mapping $\bar{F}$ given by

$$
\bar{F}(X):=\lim _{k \rightarrow \infty} F^{k}(X)=\left(\begin{array}{cc}
\overline{\mathcal{F}}_{h} & 0 \\
0 & \overline{\mathcal{F}}_{p}\left(\overline{\mathcal{F}}_{h} H\right)
\end{array}\right) X=\left(\begin{array}{cc}
\mathbf{v}_{h} \mathbf{1}_{m}^{\top} & 0 \\
0 & \mathbf{v}_{p}\left(\mathbf{v}_{h} \mathbf{1}_{m}^{\top} H\right) \mathbf{1}_{m}^{\top}
\end{array}\right) X
$$

and $\mathbf{v}_{p}(\cdot) \in C^{1}\left(\mathbb{R}_{+}^{m}\right)$

Proof. The result follows as a direct application of Proposition 1.3.1 and Lemma 1.3.4 in the abstract setting of [23] taking variables $X^{1}$ to be $H$ and $X^{2}$ to be $P$.

Let us define the global variables as the total number of host and parasitoids, i.e,

$$
h:=h^{1}+\cdots+h^{m}=\mathbf{1}_{m}^{\top} H \quad ; \quad p:=p^{1}+\cdots+p^{m}=\mathbf{1}_{m}^{\top} P .
$$

Then we can write

$$
\bar{F}(X)=E \circ G(X)
$$


where the functions $G: \mathbb{R}_{+}^{2 m} \longrightarrow \mathbb{R}_{+}^{2}$ and $E: \mathbb{R}_{+}^{2} \longrightarrow \mathbb{R}_{+}^{2 m}$ are defined by:

$$
G(X)=G(H, P)=(h, p)^{\top} \quad ; \quad E(h, p)=\left(h \mathbf{v}_{h}, p \mathbf{v}_{p}^{*}(h)\right) \quad ; \quad \mathbf{v}_{p}^{*}(h):=\mathbf{v}_{p}\left(h \mathbf{v}_{h}\right) .
$$

Therefore Hypothesis 3.2 in Section 3 holds and system (4.17) meets the conditions that allow us to reduce it. Following the general procedure of Section 3, the aggregated system is

$$
Y_{n+1}=W\left(Y_{n}\right)
$$

where

$$
Y_{n}:=G\left(H_{n}, P_{n}\right)=\left(h_{n}, p_{n}\right)^{\top} \quad ; \quad W:=G \circ S \circ E,
$$

i.e.,

$$
h_{n+1}=h_{n} \sum_{i=1}^{m} \lambda_{i} v_{i h} e^{-a_{i} v_{i p}^{*}\left(h_{n}\right) p_{n}} \quad ; \quad p_{n+1}=h_{n} \sum_{i=1}^{m} c_{i} v_{i h}\left(1-e^{-a_{i} v_{i p}^{*}\left(h_{n}\right) p_{n}}\right)
$$

Let us now study the existence and stability of fixed points for system (4.20). In order to do so we consider its associated fixed point system:

$$
h=h \sum_{i=1}^{m} \lambda_{i} v_{i h} e^{-a_{i} v_{i p}^{*}(h) p} \quad ; \quad p=h \sum_{i=1}^{m} c_{i} v_{i h}\left(1-e^{-a_{i} v_{i p}^{*}(h) p}\right) .
$$

The following result establishes the existence of trivial fixed points for system (4.20). Its proof is straightforward:

Proposition 4.4. (Trivial and semi-trivial fixed points.) Consider the fixed point equation (4.21). Then:

- $(h, p)=(0,0)=: E_{0}$ is a fixed point for $(4.20)$.

- If $\sum_{i=1}^{m} \lambda_{i} v_{i h}=1$, then $(h, p)=\left(h_{*}, 0\right)=: E_{1}\left(h_{*}\right)$ is a fixed point for $(4.20)$ for all $h_{*}>0$.

Now we are interested in finding non trivial fixed points:

Proposition 4.5. (Non-trivial fixed points.) Consider system (4.20) and assume that

$$
\sum_{i=1}^{m} \frac{\lambda_{i} v_{i h}}{\Lambda^{a_{i} /(a \delta)}}>1
$$

for certain constants $a \leq \min \left\{a_{i}\right\}, \delta \leq \min \left\{\mu_{i}\right\}$ and $\Lambda \geq \max \left\{\lambda_{i}\right\}$. Then:

1. There exists a region $\mathcal{R}=\left[\alpha_{1}, \alpha_{2}\right] \times\left[\beta_{1}, \beta_{2}\right] \subset \mathbb{R}_{+}^{2}$ which is invariant for system (4.20).

2. There exists at least one non-trivial fixed point for system (4.20) in $\mathcal{R}$

Proof. Part 1 follows as a direct application of Proposition 1.3.10 in [23]. Since $\mathcal{R}$ is an invariant, compact, convex region for the map induced by (4.20), part 2 follows from an straightforward application of the Brouwer's Fixed Point Theorem.

Let us now study the stability of the previous fixed points. The jacobian matrix $J W(H, P)$ for system $(4.20)$ in the points $E_{0}$ and $E_{1}\left(h_{*}\right)$ is found to be

$$
J W(0,0)=\left(\begin{array}{cc}
\sum_{i=1}^{m} \lambda_{i} v_{i h} & 0 \\
0 & 0
\end{array}\right), J W\left(h_{*}, 0\right)=\left(\begin{array}{cc}
1 & -h_{*} \sum_{i=1}^{m} \lambda_{i} v_{i h} a_{i} v_{i p}^{*}\left(h_{*}\right) \\
0 & h_{*} \sum_{i=1}^{m} c_{i} v_{i h} a_{i} v_{i p}^{*}\left(h_{*}\right)
\end{array}\right)
$$

Therefore $E_{1}\left(h_{*}\right)$ is not hyperbolic and so Theorem 2.6 does not apply, so we cannot guarantee either existence or stability of a corresponding fixed point in the original system (4.17). On the other hand, if $\sum_{i=1}^{m} \lambda_{i} v_{i h} \neq 1, E_{0}$ is hyperbolic and asymptotically stable (resp. unstable) if $\sum_{i=1}^{m} \lambda_{i} v_{i h}<1$ (resp. $>1$ ).

Regarding the stability of possible non-trivial fixed points for (4.19), we have not found a simple condition to establish stability. The stability conditions can be checked numerically, for example using the following criterion: if $\left(h_{*}, p_{*}\right)$ is a fixed point to (4.19) then it is asymptotically stable if, and only if,

$$
\mid \operatorname{tr}\left(J W\left(h_{*}, p_{*}\right) \mid<1+\operatorname{det}\left(J W\left(h_{*}, p_{*}\right)\right)<2 .\right.
$$

Therefore, as a direct consequence of Theorem 2.6 we have the following result that relates the dynamics of the reduced system (4.19) with those of the original system (4.17): 
Proposition 4.6. The following holds:

1. If $\sum_{i=1}^{m} \lambda_{i} v_{i h}<1$, (resp. $>1$ ) then the original system (4.17) verifies that the trivial equilibrium $(H, P)=(0,0)$ is asymptotically stable (resp unstable).

2. Let $\left(h_{*}, p_{*}\right)$ be a hyperbolic asymptotically stable (resp. unstable) fixed point of (4.19). Then there exists $k_{0} \in \mathbb{N}$ such that for all $k \geq k_{0}$ there exists a hyperbolic asymptotically stable (resp. unstable) fixed point $\left(H_{* k}, P_{* k}\right)$ for the original system (4.17). Moreover, $\left(H_{* k}, P_{* k}\right)$ converges to $\left(h_{*} \mathbf{v}_{h}, p^{*} \mathbf{v}_{p}^{*}\left(h_{*}\right)\right)$ when $k \rightarrow \infty$.

In [25] it is undertaken the numerical study of the stability of the non-trivial fixed points of system (4.19) for a certain range of parameters values. These stability conditions are compared to the rather empirical stability criterium known as $C V^{2}>1$ rule $([36])$. There is a good agrement between them which improves when the host growth rate decreases.

The expression of the aggregated model (4.19) differs from that of the Nicholson-Bailey model, which is used to represent the local interactions (4.16) in model (4.17). This is an example of the so-called functional emergence ([5]). The numerical study in [25] shows three types of asymptotic behaviour (unstability, asymptotically stable equilibrium and attracting closed invariant curve) of system (4.19) whilst the Nicholson-Bailey model always leads to unstability, what it is known as dynamical emergence ([5]).

\subsection{Nonlinear Systems III: Non matrix fast dynamics.}

The model presented in this section is a slight generalization of the one studied in [21]. We consider an agestructured population with two age classes: non reproductive (juvenile) and reproductive (adult) individuals. Reproductive individuals compete to access the available resources, food or mates. When two adults meet each of them chooses to behave aggressively (hawk tactic, $H$ ) or non-aggressively (dove tactic, $D$ ) in order to get the best payoff.

We denote the population vector at time $n$

$$
X_{n}=\left(x_{n}^{1}, x_{n}^{H}, x_{n}^{D}\right)^{\top} \in \mathbb{R}^{3}
$$

where $x_{n}^{1}$ represents juveniles, $x_{n}^{H}$ hawk adults and $x_{n}^{D}$ dove adults. According to the terminology introduced in Section 3, the population is divided into two groups, the age classes, and the adult class is divided into the subgroups of hawks and doves. The behavioural changes of adult individuals are considered faster than the demographic process.

Let us describe first the fast dynamics of the model. We suppose that adult individuals frequently encounter each other and compete for resources. Each adult encounter yields a payoff, measured in terms of gains $(G)$ and costs $(C)$. The payoff depends on the tactics adopted by each of the opponents. Individuals choose the tactic according to the conspecific they encounter (physical strength, dominance rank,...) and their experience in previous contests. Thus, each adult will play the tactic that corresponds, on average, to the best payoff. We assume that the proportion of adult individuals playing a strategy increases when the difference between the payoff of this tactic and the average payoff in the population is positive, and decreases otherwise. These hypotheses are met if we represent adult behavioural changes by means of a discrete version of the well known replicator dynamics with the payoffs corresponding to the classical hawk-dove matrix game. Such a discretization of the classical hawk-dove game is proposed in [15] (see 7.4.7) and studied in [1]. The map describing the fast dynamics for adult individuals is thus defined as

$$
F_{2}\left(x^{H}, x^{D}\right):=\left(\begin{array}{c}
\frac{-(G+C)\left(x^{H}\right)^{2}\left(x^{H}+x^{D}\right)+(2 G+C) x^{H}\left(x^{H}+x^{D}\right)^{2}}{-C\left(x^{H}\right)^{2}+(G+C)\left(x^{H}+x^{D}\right)^{2}} \\
\frac{G\left(x^{D}\right)^{2}\left(x^{H}+x^{D}\right)+C x^{D}\left(x^{H}+x^{D}\right)^{2}}{-C\left(x^{H}\right)^{2}+(G+C)\left(x^{H}+x^{D}\right)^{2}}
\end{array}\right) .
$$

As juveniles are not affected by game dynamics the fast dynamics of the model is defined by map

$$
F(X)=F\left(x^{1}, x^{H}, x^{D}\right)=\left(x^{1}, F_{2}\left(x^{H}, x^{D}\right)\right) .
$$

Demography, the slow dynamics, is driven by a map defined through a density dependent Leslie matrix of the form

$$
S\left(x^{1}, x^{H}, x^{D}\right)=\left(\begin{array}{ccc}
0 & \phi_{H}\left(x^{H}, x^{D}\right) & \phi_{D}\left(x^{H}, x^{D}\right) \\
q \sigma_{1}\left(x^{1}\right) & \sigma_{H}\left(x^{H}, x^{D}\right) & 0 \\
(1-q) \sigma_{1}\left(x^{1}\right) & 0 & \sigma_{D}\left(x^{H}, x^{D}\right)
\end{array}\right)\left(\begin{array}{l}
x^{1} \\
x^{H} \\
x^{D}
\end{array}\right)
$$


where parameter $q \in(0,1)$ represents the proportion of juveniles becoming hawk when surviving to adult (respectively, $(1-q)$ for dove). The survival rate of juveniles is $\sigma_{1}\left(x^{1}\right)$ and depends on juvenile density. The survival and fertility rates of hawk and dove adults are, respectively, $\sigma_{H}\left(x^{H}, x^{D}\right), \sigma_{D}\left(x^{H}, x^{D}\right), \phi_{H}\left(x^{H}, x^{D}\right)$ and $\phi_{D}\left(x^{H}, x^{D}\right)$, which are supposed to be density dependent too. These functions display particular features of the populations represented by the model ([11], [13]).

Finally, the proposed model coupling fast adult behavioural changes and slow Leslie-type demography reads, in the form of system (3.1), as follows

$$
X_{k, n+1}=S\left(F^{k}\left(X_{k, n}\right)\right)
$$

To proceed to the reduction of the system (4.27) notice that $F_{2}$ keeps constant the total number of adults $x^{H}+x^{D}$. Thus $F_{2}$ can be expressed in terms of the proportion of hawk adults $\nu_{H}=x^{H} /\left(x^{H}+x^{D}\right)$ and the scalar function

$$
\psi\left(\nu_{H}\right)=\frac{-(G+C) \nu_{H}^{2}+(2 G+C) \nu_{H}}{-C \nu_{H}^{2}+G+C}
$$

in the following form

$$
F_{2}\left(x^{H}, x^{D}\right)=\left(x^{H}+x^{D}\right)\left(\psi\left(\nu_{H}\right), 1-\psi\left(\nu_{H}\right)\right)^{\top} .
$$

The fact that for any $\nu_{H} \in(0,1)$

$$
\lim _{k \rightarrow \infty} \psi^{k}\left(\nu_{H}\right)=\nu_{H}^{*} \quad \text { where } \quad \nu_{H}^{*}=\left\{\begin{array}{lll}
G / C & \text { if } & G<C \\
1 & \text { if } & G \geq C
\end{array}\right.
$$

implies, for positive $x^{H}$ and $x^{D}$, that

$$
\lim _{k \rightarrow \infty} F^{k}(X)=\bar{F}(X)=\left(x^{1}, \nu_{H}^{*}\left(x^{H}+x^{D}\right),\left(1-\nu_{H}^{*}\right)\left(x^{H}+x^{D}\right)\right)^{\top}
$$

so that Hypothesis 3.1 holds.

The global variables are defined by:

$$
Y=\left(y^{1}, y^{2}\right)^{\top}:=G(X):=\left(x^{1}, x^{H}+x^{D}\right)^{\top}
$$

that is, the total number of juvenile and adult individuals, and Hypothesis 3.2 is also met since we can write:

$$
\bar{F}(X)=E(G(X)) \text { with } \quad E(Y)=E\left(y^{1}, y^{2}\right):=\left(y^{1}, \nu_{H}^{*} y^{2},\left(1-\nu_{H}^{*}\right) y^{2}\right) .
$$

The aggregated system associated to system (4.27) is

$$
\left(\begin{array}{c}
y_{n+1}^{1} \\
y_{n+1}^{2}
\end{array}\right)=\left(\begin{array}{cc}
0 & \bar{\phi}\left(y_{n}^{2}\right) \\
\sigma_{1}\left(y_{n}^{1}\right) & \bar{\sigma}\left(y_{n}^{2}\right)
\end{array}\right)\left(\begin{array}{c}
y_{n}^{1} \\
y_{n}^{2}
\end{array}\right)
$$

where

$$
\begin{aligned}
& \bar{\phi}\left(y_{n}^{2}\right):=\nu_{H}^{*} \phi_{H}\left(\nu_{H}^{*} y_{n}^{2},\left(1-\nu_{H}^{*}\right) y_{n}^{2}\right)+\left(1-\nu_{H}^{*}\right) \phi_{D}\left(\nu_{H}^{*} y_{n}^{2},\left(1-\nu_{H}^{*}\right) y_{n}^{2}\right) \\
& \bar{\sigma}\left(y_{n}^{2}\right):=\nu_{H}^{*} \sigma_{H}\left(\nu_{H}^{*} y_{n}^{2},\left(1-\nu_{H}^{*}\right) y_{n}^{2}\right)+\left(1-\nu_{H}^{*}\right) \sigma_{D}\left(\nu_{H}^{*} y_{n}^{2},\left(1-\nu_{H}^{*}\right) y_{n}^{2}\right)
\end{aligned}
$$

To apply Theorem 2.6 we need to prove that Hypothesis 3.3 holds. This is done in [21] (Appendix A) by making that the differentials of the iterates of map $F$ can be expressed in terms of the derivatives of the iterates of the scalar function $\psi$. Now, the stability of the equilibria of the general system (4.27) can be studied through system $(4.30)$.

System (4.30) is very general to obtain simple conditions for the existence and stability of positive equilibria. Further assumptions must be done to carry out this analysis. In [21], functions $\phi_{H}, \phi_{D}, \sigma_{H}$ and $\sigma_{D}$ are considered dependent on hawk and dove proportions, what entails $\bar{\phi}$ and $\bar{\sigma}$ being constant. The next simple form of the juvenile survival rate $\sigma_{1}\left(x^{1}\right)=s_{1} /\left(1+\beta x^{1}\right)$, with $s_{1}$ and $\beta$ being positive parameters, is also assumed. Under this assumptions it is proved that:

1. If $\bar{\phi} s_{1}+\bar{\sigma}<1$, then the trivial equilibrium is globally asymptotically stable for system (4.30).

2. If $\bar{\phi} s_{1}+\bar{\sigma}>1$, then system (4.30) possesses a unique positive equilibrium:

$$
E^{*}=\left(\frac{\bar{\phi} s_{1}+\bar{\sigma}-1}{\beta(1-\bar{\sigma})}, \frac{\bar{\phi} s_{1}+\bar{\sigma}-1}{\bar{\phi} \beta(1-\bar{\sigma})}\right)
$$

which is asymptotically stable. 
These asymptotic results are extended ([21], Theorem 3.1) to the complete system (4.27). It is proved that, for sufficiently large time scales ratio, i.e. large enough $k$, if $\bar{\phi} s_{1}+\bar{\sigma}<1$ the solutions of system (4.27) tend to zero, and if $\bar{\phi} s_{1}+\bar{\sigma}>1$ system (4.27) possesses an asymptotically stable positive equilibrium which can be approximated, together with its basin of attraction, by

$$
\left(\frac{\bar{\phi} s_{1}+\bar{\sigma}-1}{\beta(1-\bar{\sigma})}, \nu_{H}^{*} \frac{\bar{\phi} s_{1}+\bar{\sigma}-1}{\bar{\phi} \beta(1-\bar{\sigma})},\left(1-\nu_{H}^{*}\right) \frac{\bar{\phi} s_{1}+\bar{\sigma}-1}{\bar{\phi} \beta(1-\bar{\sigma})}\right)
$$

and the basin of attraction of $E^{*}$.

The existence of this positive equilibrium allows to analyze the influence of individual behaviour, described by game parameters, gain $G$ and cost $C$, on population fitness, represented by the total population density. The gain $G$ is assumed to measure the resource abundance and the cost $C$ the individual aggressiveness. We suppose that individuals would try to find an environment that maximizes the total population density in the long term.

If $G>C$, rich environment, the adult population is pure hawk and population fitness increases with resources availability and decreases with the cost of aggressiveness. A more interesting scenario appears when $G<C$, then for each environment richness level there is particular cost value maximizing population fitness. Assuming that population tends to adapt to this situation, we have that ([21]) increasing environment richness entails increasing population fitness and individual aggressiveness level while at the same time decreasing the fraction of aggressive individuals, $G / C$, in the population. On the other hand, in an environment becoming poorer individual would tend to be less aggressive with a larger proportion of aggressive individuals to optimize population fitness.

\section{Conclusions and perspectives.}

In this work we review the aggregation of variables method for discrete dynamical systems. These methods start from a complex system including two time scales. They consist of extracting out of the complex system a few global variables that help describe its asymptotic behaviour. For that, a reduced system that approximates the dynamics of the global variables is proposed. This abstract procedure has a more specific counterpart in ecology hierarchy theory. The up-scaling of ecological models, i.e. translating a model from a source to a larger target scale, consists of aggregating source scale variables to target scale variables and deriving the associated target scale model functions.

The importance of this process relies on the possibility of explaining and corroborating existing simpler models, as well as simplifying models in a justified way. This is also directly related to the important issue of understanding the mechanisms which are responsible for the emergence of individual behaviour at the population and community level. This question is often undertaken with the help of Individual Based Model (IBM). However, it can be difficult to obtain robust and general results from a complete and detailed IBM. In the applications we have shown how interactions between individuals can be taken into account and then, through the reduced model, how they influence the dynamics of the total population and its community. Therefore, aggregation methods can be considered as an alternative tool for the study of emergence of global properties in complex systems with many potential applications in ecological dynamics.

Some of the applications deal with the influence of spatial heterogeneity on the stability of ecological communities. New applications could also be developed in the same direction. The aggregation methods are particularly well suited for the study of spatially distributed populations in a network of patches connected by fast migrations. In this setting they provide a simpler system of equations governing the dynamics of the total populations, i.e. they show the emergence of individual migration decisions at the population level.

To finish, we propose two issues to develop from a more methodological point of view. The first one has to do with the characterization of larger classes of discrete systems to which the aggregation methods can be applied, avoiding difficult hypotheses to be proved. As a second issue, it would very useful to extend Theorem 2.6 to more general asymptotic behaviours. In this way, the aggregated system would describe to a larger extent the asymptotic behaviour of the complete system.

Acknowledgements. Authors are partially supported by: Ministerio de Ciencia e Innovación (Spain), project MTM2011-24321 (R. Bravo de la Parra, M. Marvá, E. Sánchez, L. Sanz) and Ministerio de Ciencia e Innovación (Spain), project MTM2011-25238 (R. Bravo de la Parra, M. Marvá). 


\section{References}

[1] E. Ahmed, S.A. Hegazi. On some variants of dynamical systems. Chaos Soliton. Fract., 12 (2001), No. $11,2003-2008$.

[2] P. Auger. Dynamics and thermodynamics in hierarchically organized systems. Pergamon Press, Oxford, 1989.

[3] P. Auger, R. Bravo de la Parra, J.-C. Poggiale, E. Sánchez, T. Nguyen-Huu. Aggregation of variables and applications to population dynamics. In: P. Magal, S. Ruan (Eds.). Structured Population Models in Biology and Epidemiology. Lecture Notes in Mathematics 1936, Mathematical Biosciences Subseries, Springer Verlag, Berlin, 2008 , $209-263$.

[4] P. Auger, R. Bravo de la Parra, J.-C. Poggiale, E. Sánchez, L. Sanz. Aggregation methods in dynamical systems and applications in population and community dynamics. Phys. Life. Rev., 5 (2008), No. 2, 79-105.

[5] P. Auger, J.-C. Poggiale. Aggregation and emergence in systems of ordinary differential equations. Math. Comput. Model., 27 (1998), No. 4, 1-21

[6] P. Auger, J.-C. Poggiale, E. Sánchez. A review on spatial aggregation methods involving several time scales. Ecol. Complex., 10 (2012), No. 1, 12-25.

[7] P. Auger, R. Roussarie. Complex ecological models with simple dynamics: From individuals to populations. Acta Biotheor., 42 (1994), No. 2-3, 111-136.

[8] R. Bravo de la Parra, P. Auger, E. Sánchez. Aggregation methods in discrete models. J. Biol. Syst., 3 (1995), No. 2, $603-612$.

[9] R. Bravo de la Parra, E. Sánchez, O. Arino, P. Auger. A Discrete Model with Density Dependent Fast Migration. Math. Biosci., 157 (1999), No. 1, 91-110.

[10] R. Bravo de la Parra, E. Sánchez, P. Auger. Time scales in density dependent discrete models. J. Biol. Syst., 5 (1997), No. 1, 111-129.

[11] H. Caswell. Matrix Population Models: Construction, Analysis and Interpretation, second ed. Sinauer Associates Inc., Sunderland, 2001.

[12] J.M. Cushing. An Introduction to Structured Population Dynamics. SIAM, Philadelphia, 1998.

[13] E. Dubreuil, P. Auger, J.M. Gaillard, M. Khaladi. Effect of aggressive behavior on age-structured population dynamics. Ecol. Model., 193 (2006), No. 3-4, 777-786.

[14] N. Fenichel. Persistence and Smoothness of Invariant Manifolds for Flows. Indiana U. Math. J., 21 (1972), No. 3, 193-226.

[15] J. Hofbauer, K. Sigmund. Evolutionary Games and Population Dynamics. Cambridge University Press, Cambridge, 1998.

[16] Y. Iwasa, V. Andreasen, S. Levin. Aggregation in model ecosystems I: Perfect Aggregation. Ecol. Model., 37 (1987), No. 3-4, 287-302.

[17] Y. Iwasa, S. Levin, V. Andreasen. Aggregation in model ecosystems. II. Approximate Aggregation. J. Math. Appl. Med. Biol., 6 (1989), No. 1, 1-23.

[18] P. Lett, P. Auger, R. Bravo de la Parra. Migration Frequency and the Persistence of Host-Parasitoid Interactions. J. Theor. Bio., 221 (2003), No. 4, 639-654.

[19] H. Lischke, T.J. Löffler, P.E. Thornton, N.E. Zimmermann. Up-scaling of biological properties and models to the landscape level. In: F. Kienast, S. Ghosh, O. Wildi (Eds.). A Changing World: Challenges for Landscape Research. Landscape Series 8, Springer Verlag, Berlin, 2007, 273-296.

[20] N.K. Luckyanov, Yu.M. Svirezhev, O.V. Voronkova. Aggregation of variables in simulation models of water ecosystems. Ecol. Model., 18 (1983), No. 3-4, 235-240.

[21] M. Marvá, A. Moussaoui, R. Bravo de la Parra, P. Auger. A density dependent model describing age structured population dynamics using hawk-dove tactics. J. Differ. Equ. Appl., 19 (2013), No. 6, 1022-1034.

[22] M. Marvá, E. Sánchez, R. Bravo de la Parra, L. Sanz. Reduction of slow-fast discrete models coupling migration and demography. J. Theor. Biol., 258 (2009), No. 3, 371-379.

[23] M. Marvá. Approximate aggregation on nonlinear dynamical systems. Ph.D. Thesis, Universidad de Alcalá, Spain, 2011.

[24] M.G. Neubert, H. Caswell. Density-dependent vital rates and their population dynamic consequences. J. Math. Biol., 41 (2000), No. 2, 103-121.

[25] T. Nguyen Huu, P. Auger, C. Lett, M. Marvá. Emergence of global behaviour in a host-parasitoid model with densitydependent dispersal in a chain of patches. Ecol. Complex., 5 (2008), No. 1, 9-21.

[26] T. Nguyen Huu, R. Bravo de la Parra, P. Auger. Approximate aggregation of linear discrete models with two time-scales: re-scaling slow processes to the fast scale. J. Differ. Equ. Appl., 17 (2011), No. 4, 621-635.

[27] E. Sánchez, R. Bravo de la Parra, P. Auger. Discrete Models with Different Time-Scales. Acta Biotheor., 43 (1995), No. 4, 465-479.

[28] L. Sanz, J.A. Alonso. Approximate Aggregation Methods in Discrete Time Stochastic Population Models. Math. Model. Nat. Phenom., 5 (2010), No. 6, 38-69.

[29] L. Sanz, A. Blasco, R. Bravo de la Parra. Approximate reduction of multi-type Galton-Watson processes with two time scales. Math. Mod. Meth. Appl. S., 13 (2003), No. 4, 491-525.

[30] L. Sanz, R. Bravo de la Parra. Variables aggregation in a time discrete linear model. Math. Biosci., 157 (1999), No. 1, 111-146.

[31] L. Sanz, R. Bravo de la Parra. Time scales in stochastic multiregional models. Nonlinear Anal-Real., 1 (2000), No. 1, 89-122. 
[32] L. Sanz, R. Bravo de la Parra. Time scales in a non autonomous linear discrete model. Math. Mod. Meth. Appl. S., 11 (2001), No. 7, 1203-1235.

[33] L. Sanz, R. Bravo de la Parra, E. Sánchez. Two time scales non-linear discrete models approximate reduction. J. Differ. Equ. Appl., 14 (2008), No. 6, 607-627.

[34] E. Seneta. Non-Negative Matrices and Markov Chains. Springer Verlag, New York, 1981.

[35] G.W. Stewart, J.I. Guang Sun. Matrix Perturbation Theory. Boston Academic Press, Boston, 1990.

[36] A.D. Taylor. Heterogeneity in hostparasitoid interactions: aggregation of risk and the $C V^{2}>1$ rule. Trends Ecol. Evol., 8 (1993), No. 11, 400-405. 